\title{
ON MAKING RINGS WEAKLY FINITE
}

\author{
PETER MALCOLMSON
}

\begin{abstract}
Given an associative ring $R$ with unit we construct the largest homomorphic image of $R$ such that every right invertible square matrix is left invertible. The construction may be accomplished in one step. Related results are also given.
\end{abstract}

In this note all rings are associative with unit element. Such a ring $R$ is said to be weakly finite if, for any square matrices $A$ and $B$ over $R$ such that $A B=I$ (the identity matrix), we also must have $B A=I$. The terminology is due to $\mathrm{P}$. M. Cohn $([1,0.2],[2])$, but other authors have considered this condition under other names (for example Goodearl, in [3]).

If $R$ is not weakly finite, form an ideal $J$ by including the entries of all $I-B A$ where $A B=I$ and $A$ and $B$ are square. Then $R / J$ is closer to being weakly finite, but we may have to repeat the process (possibly infinitely) to obtain finally a weakly finite homomorphic image of $R$. The purpose of this note is to show that no such repetition is necessary; that is, $R / J$ is itself weakly finite. Other results can be obtained by keeping track of the sizes of the matrices involved.

Suppose that $A$ and $B$ are $n \times n$ matrices over $R$ and that $A B=I$. We think of $A$ as a map of free right $R$-modules, and get an exact sequence

$$
0 \rightarrow P \rightarrow R^{n} \stackrel{A}{\rightarrow} R^{n} \rightarrow 0,
$$

where $P$ is the kernel of $A$. The map $B$ provides a splitting of this sequence, and the following result becomes obvious.

Proposition 1. Given a right $R$-module $P$, the following are equivalent:

(i) $P \oplus R^{n} \cong R^{n}$, where $R^{n}$ denotes the free right $R$-module of rank $\dot{n}$;

(ii) there exist $n \times n$ matrices $A, B$ over $R$ (considered as maps of free modules) such that $A B=I$ and $P \simeq \operatorname{Im}(I-B A)$.

Call a projective $P$ stably trivial of rank $<n$ if it satisfies the above condition(s), and call it stably trivial if it is stably trivial of some (finite) rank. Then clearly $R$ is weakly finite if and only if every stably trivial module is the zero module. To keep track of matrix sizes, call $R$ weakly $n$-finite (terminology of Cohn) if there are no nonzero stably trivial modules of rank $<n$. Equivalently, $R$ is weakly $n$-finite if and only if any $n \times n$ right invertible matrix over $R$ is left invertible.

Recall that the trace ideal $t(P)$ of a projective module $P$ is the ideal generated by the images of $R$-functionals $f: P \rightarrow R$. If $P$ is finitely generated, it is a direct

Received by the editors September 4, 1979 and, in revised form, November 14, 1979.

AMS (MOS) subject classifications (1970). Primary 16A50, 15A33.

Key words and phrases. Weakly finite, directly finite, stably trivial projective. 
summand of some finitely generated free $R$-module; hence $P$ is isomorphic to the image of some idempotent matrix $E$. In this case $t(P)$ is generated by the entries of $E$, or equivalently by the elements of the form $u E v$, where $E$ is (say) $n \times n, u$ is $1 \times n$ and $v$ is $n \times 1$.

The trace ideal $t(P)$ is the set of sums of such expressions. To see how sums may be expressed, suppose that projectives $P_{1}$ and $P_{2}$ are images of idempotent matrices $E_{1}$ and $E_{2}$, respectively. Then we may write

$$
u_{1} E_{1} v_{1}+u_{2} E_{2} v_{2}=\left(u_{1} u_{2}\right)\left(\begin{array}{cc}
E_{1} & 0 \\
0 & E_{2}
\end{array}\right)\left(\begin{array}{l}
v_{1} \\
v_{2}
\end{array}\right),
$$

where the right-hand side is of the form $u E v$ for $\operatorname{Im}(E) \cong P_{1} \oplus P_{2}$.

If $P_{1}$ and $P_{2}$ are stably trivial of rank $\leqslant n$, then so is $P_{1} \oplus P_{2}$, so that we might expect to do better in this addition formula. In fact, if $P_{1} \cong \operatorname{Im}\left(I-B_{1} A_{1}\right)$ and $P_{2} \cong \operatorname{Im}\left(I-B_{2} A_{2}\right)$ with $A_{1} B_{1}=I=A_{2} B_{2}($ all $n \times n)$, then

$$
\begin{aligned}
& u_{1}\left(I-B_{1} A_{1}\right) v_{1}+u_{2}\left(I-B_{2} A_{2}\right) v_{2} \\
& \quad=\left(u_{1} A_{2}+u_{2}\left(I-B_{2} A_{2}\right)\right)\left(I-B_{2} B_{1} A_{1} A_{2}\right)\left(B_{2} v_{1}+\left(I-B_{2} A_{2}\right) v_{2}\right)
\end{aligned}
$$

and $\left(A_{1} A_{2}\right)\left(B_{2} B_{1}\right)=I$. Thus the sum is expressed without increase in the size of the idempotent matrix. This computation allows us to prove the following lemma.

LeMma 1. Suppose $P$ is a finitely generated projective and $X$ is a matrix with entries in $t(P)$. Then $X$ may be written in the form $X=U E V$, where $E$ is an idempotent matrix whose image is a direct sum of copies of $P$. Furthermore, if $P$ is stably trivial of rank $\leqslant n$, then $E$ may be chosen to be $n \times n$ and of the form $I-B A$, where $A B=I$.

Proof. By using the addition formulae above, there is no trouble if $X$ is $1 \times 1$ or even if $X$ has only one nonzero entry, by appropriate choice of $U$ and $V$. But we may use the formulae again to obtain any $X$ by adding together matrices with one nonzero entry. In the stably trivial case this may be accomplished without increasing the size of the idempotent matrix.

Another calculation provides us with the following fundamental lemma.

LEMMA 2. Let $P$ be stably trivial over $R$ of rank $\leqslant n$ and suppose $\bar{Q}$ is stably trivial over $R / t(P)$ of rank $\leqslant n$. Then there exists $Q$ stably trivial over $R$ of rank $<n$ such that $Q \otimes R / t(P) \cong \bar{Q}$.

Proof. Let $\bar{Q} \cong \operatorname{Im}(I-\overline{B A})$, where $\bar{A}$ and $\bar{B}$ are $n \times n$ matrices over $R / t(P)$ such that $\overline{A B}=I$. Lift $\bar{A}, \bar{B}$ to matrices $A, B$ over $R$; then $I-A B$ has entries in $t(P)$. By Lemma 1 we may find $n \times n$ matrices $X, Y, U, V$ over $R$ such that $X Y=I$ and $I-A B=U(I-Y X) V$. Furthermore, $\operatorname{Im}(I-Y X)$ is isomorphic to a direct sum of copies of $P$, so each entry in $I-Y X$ is in $t(P)$. Now put $Z=A X+U(I-Y X)$ and $W=Y B+(I-Y X) V$; then $Z W=I$. Thus we may put $Q=\operatorname{Im}(I-W Z)$, stably trivial over $R$ of rank $\leqslant n$. If we denote by $\bar{X}$ and $\bar{Y}$ the images of $X$ and $Y$ over $R / t(P)$, then $Q \otimes R / t(P) \cong \operatorname{Im}(I-\overline{Y B A X}) \cong$ $\operatorname{Im}(I-\overline{B A}) \cong \bar{Q}$.

Recall that the trace ideal of a direct sum of projectives is the sum of the 
corresponding trace ideals. Furthermore, if $f: R \rightarrow S$ is a ring homomorphism and $P$ is a projective $R$-module, then $f(t(P)) \subseteq t(P \otimes S)$. If $P$ is stably trivial over $R$, then $P \otimes S$ is stably trivial over $S$.

Now for any ring $R$, denote by $S T_{n}(R)$ the direct sum of all stably trivial direct summands of $R^{n}$. The following is our main result.

THEOREM 1. Let $J_{n}=t\left(S T_{n}(R)\right)$. Then $R / J_{n}$ is weakly $n$-finite. Furthermore, any ring homomorphism from $R$ to a weakly $n$-finite ring factors through $R / J_{n}$.

Proof. Suppose $\bar{Q}$ is a stably trivial module over $R / J_{n}$ of rank $\leqslant n$. Then there are $n \times n$ matrices $\bar{A}, \bar{B}$ over $R / J_{n}$ with $\overline{A B}=I$ and $\bar{Q} \cong \operatorname{Im}(I-\overline{B A})$. Lifting $\bar{A}$, $\bar{B}$ to matrices $A, B$ over $R$, we see that $I-A B$ has entries in $J_{n}$, hence in some finite sum of trace ideals of stably trivial modules of rank $\leqslant n$. Any such finite sum is the trace ideal of a single stably trivial module $P$ of rank $\leqslant n$. Thus $I-A B$ has entries in $t(P) \subseteq J_{n}$, so over $R / t(P)$ there is a stably trivial $Q^{\prime}$ with $Q^{\prime} \otimes R / J_{n} \approx$ $\bar{Q}$. But by Lemma $2 Q^{\prime}$ lifts to a stably trivial module $Q$ over $R$ of rank $<n$ such that $Q \otimes R / t(P) \cong Q^{\prime}$. By construction of $S T_{n}(R)$ we have $t(Q) \subseteq J_{n}$, so that $\underline{Q} \otimes R / J_{n}=0$. But $Q \otimes R / J_{n} \cong Q \otimes R / t(P) \otimes R / J_{n} \cong Q^{\prime} \otimes R / J_{n} \cong \bar{Q}$. Thus $\bar{Q}=0$ and $R / J_{n}$ is weakly $n$-finite. Given any ring homomorphism $R \rightarrow T$ where $T$ is weakly $n$-finite, we need to show that $J_{n}$ is contained in the kernel. But $J_{n}$ is mapped into $t\left(S T_{n}(T)\right)=0$.

Categorically, we may say that the full subcategory of weakly $n$-finite rings is reflective in the category of all rings. The case of weakly finite rings is done similarly; again for any $R$, let $S T(R)$ be the union of the $S T_{n}(R)$ for all $n$.

COROLlary 1. Let $J=t(S T(R))$. Then $R / J$ is weakly finite. Furthermore, any ring homomorphism from $R$ to a weakly finite ring factors through $R / J$.

Proof. A stably trivial module $\bar{Q}$ over $R / J$ of rank $\leqslant k$ may be lifted to a stably trivial module over $R / t(P)$ of rank $\leqslant k$, where $P$ is stably trivial over $R$ of rank $\leqslant m$ (say), by following the proof of Theorem 1. Putting $n=\max (k, m)$, we complete the proof as for Theorem 1 .

In some cases the ring $R / t(S T(R))$ must be the zero ring, as for example when some free module is stably trivial (i.e., the ring does not have invariant basis number [1]). Recall that a projective module $P$ is a generator if the free module $R$ is a direct summand of a direct sum of copies of $P$, and that this occurs if and only if $t(P)$ is the unit ideal. Then we have the following equivalences.

Profosition 2. For any ring $R$ the following are equivalent:

(a) $t\left(S T_{n}(R)\right)=R$;

(b) the identity matrix $I_{n+1}$ is a product of an $(n+1) \times n$ and an $n \times(n+1)$ matrix;

(c) the free module $R$ is a direct summand of some stably trivial module of rank $\leqslant n$.

Proof. To prove (a) implies (b), assume $1 \in t\left(S T_{n}(R)\right)$. Then 1 is in some finite sum of trace ideals, hence in $t(P)$ for some stably trivial $P$ of rank $\leqslant n$. By Lemma 
1 we have $1=u(I-B A) v$, where $u$ is $1 \times n$, $v$ is $n \times 1$ and $A B=I_{n}$. Thus

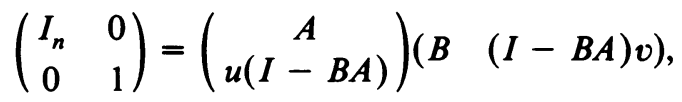

proving (b). Assuming (b), we get a split surjection of free modules $R^{n} \rightarrow R^{n+1}$. Denote the kernel by $K$; then $K \oplus R^{n+1} \cong R^{n}$ and $K \oplus R$ is stably trivial of rank $<n$, proving (c). Finally, (c) certainly implies that $S T_{n}(R)$ is a generator, giving (a).

There is an obvious analogous result for $S T(R)$. The analogue of condition (b) (that some identity matrix is "nonfull") is considered by Cohn in [2].

Note that the basic result in Lemma 2 shows that stably trivial modules over $R / t(P)$ may be lifted, but says nothing about arbitrary projectives. Thus it is possible that $R / t(P)$ might have "new" projectives, not induced from $R$. In particular if $R$ is taken with (noncommuting) generators $x, y, u, v, e$ and relations $x y=1, e-e^{2}=u(1-y x) v$, then the image of $e$ in $R / S T(R)$ is likely to give a new projective. However, this example seems too complicated to check.

However, the methods of Lemma 2 at least provide the following isolated result.

Proposition 3. Let $P$ be a finitely generated projective over $R$ and suppose $\bar{Q}$ is stably trivial over $R / t(P)$. Then there is a finitely generated projective $Q$ over $R$ such that $\bar{Q} \cong Q \otimes R / t(P)$.

Proof. We get matrices $\bar{A}, \bar{B}$ over $R / t(P)$ with $\overline{A B}=I$ and $\bar{Q} \cong \operatorname{Im}(I-\overline{B A})$. Lift $\bar{A}, \bar{B}$ to $A, B$ over $R$; then $I-A B$ has entries in $t(P)$. By Lemma 1 we may find matrices $U, E, V$ over $R$ with $I-A B=U E V$ and $E$ idempotent with entries in $t(P)$. Now put

$$
F=\left(\begin{array}{cc}
E-E V U E & -E V A \\
-B U E & I-B A
\end{array}\right)
$$

then $F^{2}=F$. Put $Q=\operatorname{Im}(F)$, projective over $R$; then

$$
Q \otimes R / t(P) \cong \operatorname{Im}\left(\begin{array}{cc}
0 & 0 \\
0 & I-\overline{B A}
\end{array}\right) \simeq \bar{Q} .
$$

\section{REFERENCES}

1. P. M. Cohn, Free rings and their relations, Academic Press, London, 1971.

2. __ Some remarks on the invariant basis property, Topology 5 (1966), 215-228.

3. K. R. Goodearl and D. Handelman, Rank functions and $K_{0}$ of regular rings, J. Pure Appl. Algebra 7 (1976), 195-216.

Department of Mathematics, Wayne State University, Detrort, Michigan 48202 